\title{
Dynamic Changes with Liver Atrophy as an Alternate Marker of Chemotherapy-Associated Liver Injury
}

\author{
Toshifumi Wakai, MD, PhD, FACS ${ }^{1}$, and Pankaj Prasoon, MD, PhD ${ }^{1}$ \\ Division of Digestive and General Surgery, Niigata University Graduate School of Medical and Dental Sciences, Niigata \\ City, Japan
}

Recently, an increasing number of patients with colorectal liver metastases (CLM) have become possible candidates for surgery after comprehensive and efficient chemotherapy. However, for patients who have CLM with steatohepatitis, the chemotherapy regimen should be carefully considered because the risk of hepatotoxicity is significant. ${ }^{1}$ With the greater likelihood of liver injury after extensive chemotherapy, assessment of the underlying liver and surgery decisions dependent on dynamic volumetric data have become essential. ${ }^{2,3}$

The article by Shindoh et al. ${ }^{4}$ published in Annals of Surgical Oncology represents yet another significant influence from their group that increases our knowledge of liver atrophy associated with chemotherapy. In this impressive clinical study by the authors, an ideal attempt was made to gauge the significance of liver atrophy during chemotherapy for colorectal cancer. The authors explored the kinetic change in liver parenchymal volume during chemotherapy for colorectal cancer and determined that liver atrophy is associated with hepatic function reserve. Liver atrophy is associated with refractory ascites, pleural effusion, and sinusoidal injuries.

The current study demonstrated the significance of liver atrophy as the alternative marker of chemotherapy-associated liver injury. It showed a robust correlation between sinusoidal injury in the normal liver parenchyma and the degree of liver atrophy. These particular components after chemotherapy are acknowledged, and it is important for the

(C) Society of Surgical Oncology 2019

First Received: 4 July 2019;

Published Online: 19 August 2019

T. Wakai, MD, PhD, FACS

e-mail: wakait@med.niigata-u.ac.jp clinical setting to understand the predictive variables of liver atrophy before chemotherapy. In the foreseeable future, study analyzing the potential risk for progression of liver atrophy and sinusoidal injury before chemotherapy would provide considerable details relevant to chemotherapy-associated liver injury.

In multivariate analyses, the authors noted the influence of antibodies against epidermal growth factor receptor (EGFR) in 18 patients who underwent anti-EGFR therapy. Nevertheless, the anti-EGFR antibodies had no influence on liver atrophy, sinusoidal injury, or postoperative complications. This outcome is fascinating, and a high-volume study is required to validate the result. An additional significant outcome was that the degree of liver atrophy seemed to be an effective predictor of the hepatic functional reserve, although the correlation was slight. A propensity toward an impaired indocyanine green retention rate at $15 \mathrm{~min}$ (ICG-R15) was verified with the escalating degree of atrophy.

Shindoh et al. ${ }^{4}$ showed that by preventing liver atrophy or sinusoidal injury, postoperative morbidity could be reduced, which is linked to the kinetic changes in liver volume but unrelated to the superiority of bevacizumab for patients with CLM. Martins et al. ${ }^{5}$ documented that bevacizumab may protect against liver atrophy, which is associated with impaired hepatic functional reserve and increased morbidity. The evaluation used a prospectively collected database of patients treated in the same manner throughout the study period. The patients in the validation cohort were from two patient groups undergoing chemotherapy without liver resection.

The current study by Shindoh et al. ${ }^{4}$ concluded that liver atrophy during or after chemotherapy shows impaired hepatic functional reserve as well as a higher risk of postoperative morbidity and hepatic dysfunction during 
and after chemotherapy. Bevacizumab may decrease the risk for the development of liver atrophy, probably by preventing sinusoidal injury. Further studies on this protective effect of bevacizumab are warranted.

\section{REFERENCES}

1. Vauthey JN, Pawlik TM, Ribero D, et al. Chemotherapy regimen predicts steatohepatitis and an increase in 90-day mortality after surgery for hepatic colorectal metastases. J Clin Oncol. 2006;24:2065-72.

2. Ribero D, Abdalla EK, Madoff DC, et al. Portal vein embolization before major hepatectomy and its effects on regeneration, resectability, and outcome. Br J Surg. 2007;94:1386-94.

3. Shindoh J, Truty MJ, Aloia TA, et al. Kinetic growth rate after portal vein embolization predicts posthepatectomy outcomes: toward zero liver-related mortality in patients with colorectal liver metastases and small future liver remnant. J Am Coll Surg. 2013;216:201-9.

4. Shindoh J, Kobayashi Y, Kinowaki K, et al. Dynamic changes in normal liver parenchymal volume during chemotherapy for colorectal cancer: liver atrophy as an alternate marker of chemotherapy-associated liver injury. Ann Surg Oncol. 2019. h ttps://doi.org/10.1245/s10434-019-07740-x.

5. Martins J, Alexandrino H, Oliveira R, et al. Sinusoidal dilation increases the risk of complications in hepatectomy for CRCLM: protective effect of bevacizumab and diabetes mellitus, serum gamma-glutamyltranspeptidase as predictive factor. Eur J Surg Oncol. 2016;42:713-21.

Publisher's Note Springer Nature remains neutral with regard to jurisdictional claims in published maps and institutional affiliations. 